\title{
„Einfach hinnehmen“ - Partizipation bei der Leistungsbewertung
}

\author{
Silke Marchand $(\mathbb{D} \cdot$ Juliane Spiegler $\mathbb{D}$
}

Eingegangen: 31. Oktober 2020 / Angenommen: 7. April 2021 / Online publiziert: 9. Juli 2021

(C) Der/die Autor(en) 2021

Zusammenfassung Partizipation von Schülerinnen und Schülern gilt als wichtiges Element pädagogischen Handelns in der Demokratie, erweist sich aber in der Umsetzung als herausfordernd, da zwischen Lehrpersonen und Schülerinnen bzw. Schülern ein unaufhebbar asymmetrisches Verhältnis besteht. Es kommt in diesem Rahmen zu Partizipationssituationen, in denen die Autonomieansprüche, die mit der Forderung nach Partizipation verbunden sind, nicht eingelöst werden, auch wenn der Anschein erweckt wird, Mitbestimmung wäre möglich. Eine praxistheoretische Analyse einer Unterrichtsepisode zur Leistungsrückmeldung und -bewertung zeigt, wie die Praktiken des, doing student" und ,doing participation' ineinander verschränkt sind und dazu beitragen, eine wirkmächtige Ordnung zu etablieren, in der oberflächlich Meinungsäußerungen möglich, aber inhaltliche Widerspruchsmöglichkeiten verwehrt sind. Die als partizipativ inszenierte Bewertung ihrer Leistung sollen die Schülerinnen und Schüler „einfach hinnehmen“.

Schlüsselwörter Partizipation · Pseudo-Partizipation · Praxistheorie · Doing student $\cdot$ Doing participation

Dr. Silke Marchand $(\bowtie) \cdot$ Juliane Spiegler

Humanwissenschaftliche Fakultät, Department Erziehungs- und Sozialwissenschaften,

Arbeitsbereich Schulforschung mit dem Schwerpunkt Unterrichtstheorien und Schulsystem,

Universität zu Köln, Triforum, Albertus-Magnus-Platz, 50923 Köln, Deutschland

E-Mail: silke.marchand@uni-koeln.de

Juliane Spiegler

E-Mail: juliane.spiegler@uni-koeln.de 


\section{"Just accept"—Participation in an assessment situation}

Abstract Student participation is deemed important in pedagogical situations in a democracy, but proves to be difficult to implement due to the asymmetrical relationship between teachers and students. This leads to situations that pretend to be participatory, but do not grant autonomy. A practice theory based analysis of a teaching episode for performance feedback and assessment in an elementary classroom shows how the practices of "doing student" and "doing participation" are intertwined. Both contribute to establishing an order in which students can superficially express their opinions, but cannot contradict the teacher effectively. Although the evaluation of their performance is staged as participatory, the students are expected to "just accept" it.

Keywords Participation - Pseudo-participation - Practice theory · Doing student · Doing participation

\section{Partizipation in der Schule zwischen Autonomie und Heteronomie}

Der Partizipationsbegriff wird sowohl mit dem emanzipatorischen Fokus auf die aktive Mitgestaltung oder -entscheidung über Verhältnisse als auch mit dem sozialintegrativen Fokus auf den Zugang zu Ressourcen wie Unterricht verwendet (z. B. Reisenauer 2020, S. 3). Partizipation wird in pädagogischen Kontexten mit unterschiedlichen Begründungen (z. B. Sauerwein 2019; Reisenauer 2020) als wichtiges Element pädagogischen Handelns in der Demokratie präsentiert, auch und gerade in der Schule (KMK 2018, S. 5). Aus didaktischer Perspektive ist zu fragen, welche Ansätze besonders geeignet scheinen, dieses Postulat (vgl. Gruschka 2003, S. 74) zu befolgen, wobei z.B. demokratiepädagogische Ansätze das persönliche Erleben von Partizipation im direkten sozialen Umfeld in den Mittelpunkt stellen (Hahn-Laudenberg 2017, S. 37). Die Schule ist einerseits wegen der allgemeinen Schulpflicht aussichtsreich für Partizipationsbemühungen, weil dort alle Kinder und Jugendlichen erreicht werden (z. B. Billis 2020, S. 368). Andererseits sind die Teilnahmepflicht und feststehende Curricula problematisch für demokratische Beteiligung (Sauerwein 2019, S. 439), Erziehung zur (partizipativen) Demokratie scheint teils den Funktionen und Strukturen der Schule als Institution zu widersprechen (z. B. Billis 2020, S. 371). Das generell asymmetrische Verhältnis von Erwachsenen und Kindern gilt auch, von der Bewertungssituation verschärft, für Lehrpersonen und Schülerinnen bzw. Schüler (z. B. Feichter 2020, S. 33). Die Mitbestimmungsmöglichkeiten für Schülerinnen und Schüler in der Schule sind rechtlich begrenzt (Coelen 2010, S. 46). Trotzdem ist der Anspruch verbreitet, Partizipation in der Schule umzusetzen (vgl. für verschiedene Begründungslinien z. B. Reisenauer 2020, S. 6 ff.). Demokratische und/oder partizipative Unterrichts- oder Leistungsbewertungskonzepte wie z.B. Lernen ohne Noten (Beutel und Pant 2020) oder Portfolios (Häcker 2011) werden kaum in Bezug zu Strukturbedingungen gesetzt, die als hinderlich und widersprüchlich diskutiert werden (vgl. z.B. Betz et al. 2010; Budde 
2010; Coelen 2010; Feichter 2020; Gamsjäger et al. 2013; Helsper und Lingkost 2013 oder Reichenbach 2006).

Empirisch zeigen Partizipationssituationen in der Schule, dass Schülerinnen und Schüler nicht gleichberechtigt, sondern durch verordnete Mitbestimmung (Budde 2010, S. 387, S. 397) aufgefordert sind, sich ,in den von Erwachsenen abgesteckten Bahnen“ (Betz et al. 2010, S. 18) zu beteiligen. Dass der Rahmen ohne Aushandlung gesetzt ist (Pigorsch und Rothmaler 2020, S. 242), ist kaum zu beanstanden, denn der Anspruch an demokratische Partizipation von Kindern ist nicht, dass sie frei entscheiden können, sondern dass die Rechte und Pflichten aller Beteiligten in Entscheidungsverfahren bewusst geregelt sind (Knauer et al. 2016, S. 36). Problematisch ist, dass die Entscheidung, (nicht) zu partizipieren keine freiwillige ist (wie z.B. bei Maywald 2016, S. 27 gefordert), wenn Partizipation zur Aufgabe im Unterricht wird. Zudem müssten Möglichkeiten und Grenzen der Mitbestimmung schon vor einer Entscheidung für die Beteiligten klar sein (Reinhardt 2010, S. 90), ohne existierende Machtverhältnisse zu verschleiern (Knauer et al. 2016, S. 34).

In schulischen Kontexten sind ,die Aushandlungen tatsächlich nicht ergebnisoffen“ (Budde 2010, S. 397). Die Grenzen von Partizipation zu „Pseudo-Partizipation“ (Reichenbach 2006, S. 58) oder „Nicht-Partizipation“ (Hart 1992, S. 9) werden überschritten, wenn als Ergebnis eines vorgeblich gemeinsamen Entscheidungsprozesses nur ein im Vorfeld gefälltes Urteil in Frage kommt (Reichenbach 2006, S. 58), oder wenn Kinder sich zwar äußern dürfen/sollen, aber maximal geringe Möglichkeiten bestehen, die eigene Meinung zu vertreten (Hart 1992, S. 9 f.). Die schulischen Bedingungen bringen eine „Ambivalenz der Partizipation“ (Reichenbach 2006, S. 59) hervor, in der autonome und heteronome Elemente ineinander verwoben sind (Helsper und Lingkost 2013, S. 153). Partizipation in der Schule lässt sich also als Dimension weder quantitativ noch normativ adäquat ordnen.

Dieser Beitrag ergänzt die Diskussion um widersprüchliche Bedingungen um die Verschränkung von Partizipationspraktiken hinsichtlich normativer Anforderungen des Schülerjobs (Breidenstein 2006) bei Leistungsbewertungen (z. B. Rabenstein et al. 2013) sowie um deren subjektivierende Effekten (z. B. Ricken 2018).

Der vorliegende Text blickt mit einem praxistheoretischen Verständnis von Partizipation auf eine Unterrichtsepisode der Leistungsrückmeldung und -bewertung. Die Leistungsbewertung ist eine der Aufgaben von Lehrpersonen, in denen das asymmetrische Verhältnis zwischen ihnen und ihren Schülerinnen und Schülern besonders deutlich wird, obwohl manche Bewertungs- und Beurteilungsansätze versuchen, das zu ändern, in dem sie Lernende in den Bewertungs- und Beurteilungsprozess einbeziehen (Reinhardt 2010, S. 100). Aus praxistheoretischer Perspektive ist die Leistung nicht objektiv messbar, sondern eine soziale Konstruktion, die ,vor Dritten [...] legitimierbar“" (Idel und Rabenstein 2016, S. 13) ist. Konstruiert wird Leistung in pädagogischen Praktiken, bei denen Idel und Rabenstein (2016, S. 12) „Praktiken der Aufgabenstellung, Praktiken der Aufführung von Leistung und Praktiken der Bewertung von Leistung“ unterscheiden, während Meier (2011, S. 99) Orientierungs-, Kontroll-, Management-, Image- und Kulanzpraktiken beschreibt, die von Lehrpersonen sowie Schülerinnen und Schülern genutzt werden, wenn sie z. B. Notenbegründungen geben, formale Voraussetzungen kontrollieren, Fehler korrigieren, zeigen, was sie anerkennen oder wenn sie milder bewerten als möglich wä- 
re. Klassenöffentlich vergebene Noten werden von der Lehrperson und der Klasse in einem „kooperative[m] Zusammenspiel“ (Meier 2011, S. 112) legitimiert, auch in einem Ablauf, der nicht auf Mitbestimmung der Schülerinnen und Schüler setzt. Sie sind also am Prozess des Hervorbringens von Leistung beteiligt, inwiefern sie durch Partizipation aber (mit-)entscheiden können und an Autonomie gewinnen, ist unklar.

\section{Methodologische und methodische Überlegungen}

Das Datenmaterial entstand im Rahmen eines Dissertationsprojektes, in dem Grundschulunterricht für acht Monate ethnographisch (vgl. Breidenstein et al. 2020) erhoben wurde. Der Unterricht wurde mit der Teilnehmenden Beobachtung begleitet und audiographiert, sodass es sich bei allen Äußerungen um wortwörtlich Gesprochenes ${ }^{1}$ handelt. Methodologisch werden Praktiken als typisierte und routinisierte Aktivitätenbündel (Reckwitz 2008, S. 112) angenommen. Unterricht ist aus dieser Perspektive eine Abfolge spezifischer Praktiken, die ,,auf zugrunde liegende Strukturierungen und Ordnungen [verweisen], da die Praktik (und nur genau diese) ausschließlich dann funktioniert, wenn sie relativ reibungslos in den sozialen Vollzug“ (Budde 2014, S. 134) passt. In diesem Vollzug werden soziale Ordnungen hervorgebracht, stabilisiert, aber auch irritiert und abgewandelt (Schäfer 2013). In Bezug auf Unterricht ist diese Ordnung immer eine Leistungsordnung (Rabenstein et al. 2013), in der ein bestimmtes Wissen erzeugt wird, wie Leistungen einzuschätzen sind und was zu tun ist, um als leistungserfolgreich anerkannt zu werden (vgl. Idel und Rabenstein 2016, S. 11). Teil dieser hervorgebrachten Ordnungen sind Subjektpositionierungen, da Akteurinnen und Akteure in und durch Praktiken Positionen ein- bzw. annehmen sowie zuweisen. Dabei ist das Angesprochenwerden zentral, denn erst dadurch werden Subjekte zu Subjekten. Im (An)Sprechen sind die Akteurinnen und Akteure gleichzeitig Adressierende und Adressierte (Butler 1998, S. 53), so lässt sich Subjektivierung relational als (Re-)Adressierung auffassen. Sie ist zudem von „Normen eingerahmt" (Butler 2002, S. 144), denen man sich - sie zugleich hervorbringend unterwerfen muss.

Partizipation lässt sich somit als soziale Praxis verstehen, die erst im Vollzug bestimmter Praktiken hergestellt wird. Mit der von Pigorsch und Rothmaler (2020) vorgeschlagenen Justierung auf ,,ein doing participation als soziomaterielle und diskursive Praxis“ (S. 245, Herv. i. Orig.), wird deutlich, wie ,alltägliche Praktiken zu ,Partizipationsprozessen “ und soziale Akteur*innen zu ,Partizipand*innen“ gemacht werden“ (ebd.). Es geht also nicht darum, die didaktische Qualität des Handelns zu hinterfragen oder zu bewerten, denn aus praxeologischer Sicht interessiert das implizite Wissen um die Handlungsmöglichkeiten im lokalen Kontext (vgl. Breidenstein et al. 2011, S. 26f.). Damit wird eines der von Abs und Moldenhauer (2021,

\footnotetext{
1 Transkriptionssystem: (.)= Pause, Anzahl der Sekunden; Großbuchstaben=LAUTES SPRECHEN; — unvollständige Äußerung, die unterbrochen wird; [-] inhaltliche Kürzungen, die den Sinn der Aussage jedoch nicht verändern.
} 
S. 13) eruierten Desiderate der qualitativen Forschung zur Partizipation in Schule und Unterricht aufgegriffen.

Das analysierte Datenmaterial kombiniert die Darstellung der räumlichen Anordnung, ein Beobachtungsprotokoll sowie das Transkript der Unterrichtssequenz. Aus den ethnographischen Beobachtungen des Projekts wird eine Unterrichtsstunde fokussiert, die im Kontext anderer beobachteter Leistungssituationen und vor der Folie des skizzierten Forschungsstandes typisch erscheint. Die Methode erlaubt keine Aussagen zur Repräsentativität des Fallmaterials, kann aber für die Strukturen und Widersprüche sensibilisieren, mit denen bei Partizipation in schulischen Leistungsbewertungssituationen gerechnet werden muss.

Die Rekonstruktion zeigt auf, welche Partizipationsmöglichkeiten und -grenzen wie eröffnet und aufgegriffen werden, welche Normen den Praktiken zugrunde liegen und welche subjektivierenden Folgen in diesen Partizipationspraktiken erkennbar sind und geht so, auf die Logik des Feldes bezugnehmend, über eine Einzelfallanalyse hinaus.

\section{Fallmaterial und Rekonstruktion}

Zur Situation: Die Schülerinnen und Schüler einer dritten Klasse einer Grundschule hatten die Aufgabe, in Tandemarbeit ein Buch zu lesen und gemeinsam dessen Inhalt sprachlich-zusammenfassend sowie künstlerisch aufzuarbeiten. Die Lehrerin Frau C. hatte ein Arbeitsblatt ausgegeben, auf dem verbindliche Elemente der Buchvorstellung festgehalten waren, wie zum Beispiel Angaben zu Autorenschaft, Verlag, Hauptfiguren und das Vorlesen der spannendsten Stelle. Die Tandems erarbeiteten ihre Buchvorstellung zu einem selbstgewählten Buch über mehrere Unterrichtsstunden und präsentierten nun die Ergebnisse. Präsentation und Bewertung verliefen jeweils nach dem gleichen Schema wie in der nachfolgend geschilderten Episode.

Die räumliche Anordnung sieht wie folgt aus (Abb. 1):

Situative Ordnungsbildung ist von der räumlichen Anordnung der Akteurinnen und Akteure geprägt, durch die sich Handlungsoptionen eröffnen oder verschließen. Frau C. überlässt den Präsentationsbereich vor der Tafel den Präsentierenden, kann aber selbst alle Schülerinnen und Schüler sehen. Sie positioniert sich weder als Teil der Gruppe noch als Teil der Präsentierenden. Während ein Platz auf den Bänken eine gleichberechtig(ter)e Positionierung andeuten könnte, ließe sich ein Platz hinter der Gruppe als eine überwachende Position deuten (vgl. Breidenstein 2004). Dank ihrer Platzwahl ,ist die kontrollierende Beobachtung durch die Lehrerin selbst wiederum beobachtend kontrollierbar" (ebd., S. 94). In der Anordnung verbinden sich autonomiegewährende und kontrollierende Elemente, es entstehen drei zentrale Schauplätze und für die Präsentierenden zwei relevante Publikumsbereiche.

Applaus setzt ein. Pia und Tim bleiben vorne stehen. Frau C. fragt, ob jemand noch Fragen hat, es ist kurz still, niemand meldet sich und jemand ruft: „Keine.“ Die Lehrerin fragt daraufhin mit Blick in die Klasse: „Was war gut, top?“

Ein Mädchen fängt an zu reden: „Dass ihr immer ähm das mit Fragen.“ und Tim ruft: „Ja“ Auch Frau C. bestätigt dies und sagt: „Das war gut.“, während 


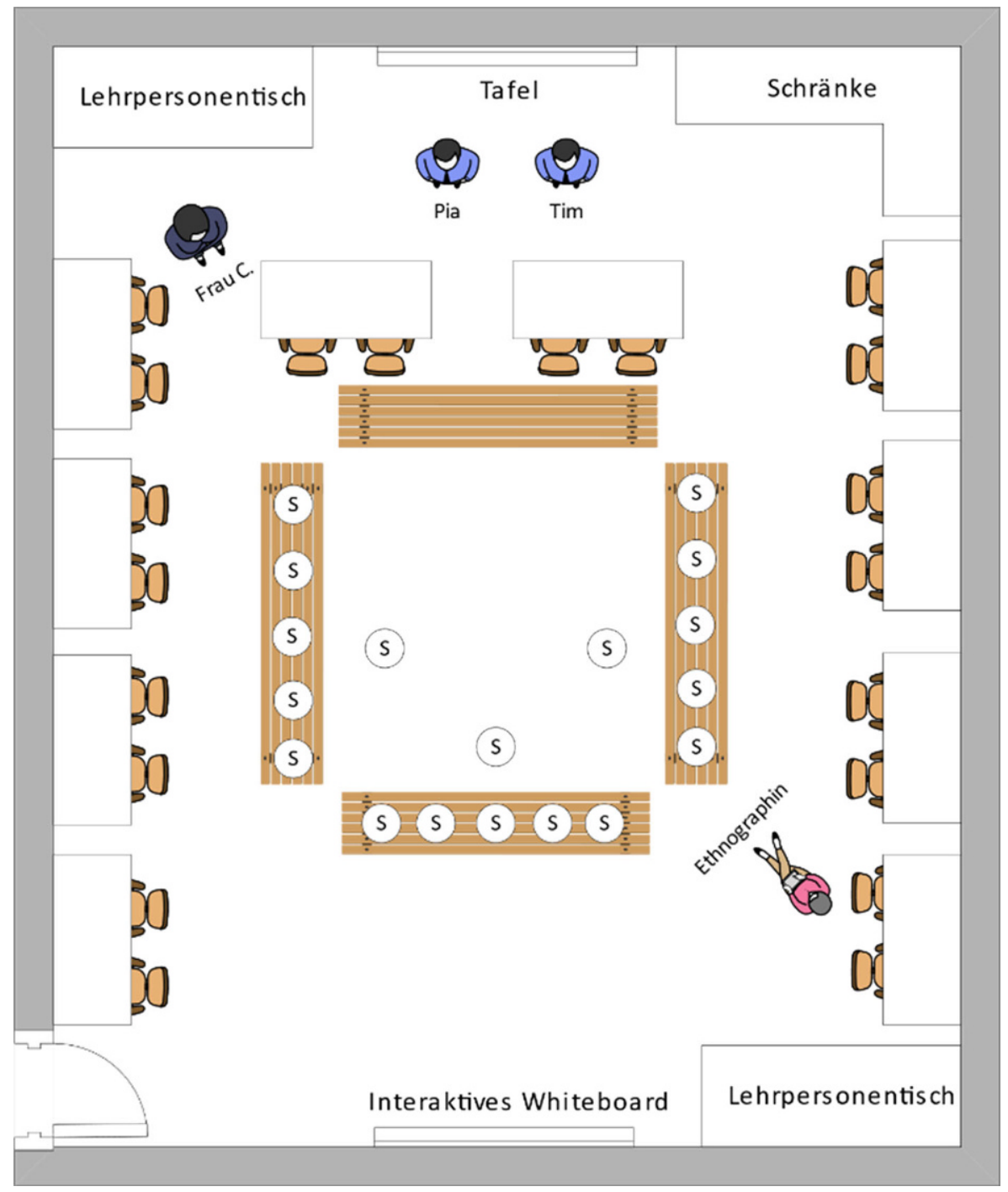

Abb. 1 Räumliche Anordnung während der Episode (eingekreistes $S=$ Schüler bzw. Schülerin)

die Schülerin noch weiterredet, man sie aber kaum versteht. Tim ruft Chris auf, der den gleichen Punkt positiv hervorhebt. Pia unterbricht Chris, indem sie Marlene aufruft, die das von Chris Gesagte bestätigt und sagt, dass sie weiterhin gut finde, was Tim abgepaust hat. Tim unterbricht sie und will anscheinend klarstellen, dass er das Bild nicht (wie die Gruppe zuvor) abgepaust, sondern selbst gezeichnet hat, doch Frau C. macht „Psst““ und schüttelt leicht den Kopf. Tim ruft Ricardo auf, der sagt: „Ich find das Bild schön.“ Pia ruft derweil „Luise!“ Da sich zwei Schülerinnen gemeldet haben, die beide Luise heißen und fast zusammensitzen, führt dies zu Irritation, eine der beiden sagt: „Du hast 
zu uns beide geguckt." Pia kichert. Frau C. dreht den Kopf zu Pia und fragt: „Und welche meinste?“ Doch als Pia antwortet: „Ähm, ihr könnt euch abwechseln.“, fordert die Lehrerin sie auf: „OCH NEE, sag jetzt eine, ZACK.“ Dem kommt Pia nach, beide Mädchen geben ihre positive Kritik ab und Tim greift eine der Antworten auf: „Das haben wir vergessen, RITT durch den WILDEN WESTEN“ Auch Pia steigt ein: „Naja, eigentlich sollte das so sein [Hält das Bild hinter die gebastelten Pappmaché-Pferde], aber ich habs vergessen und [dann weiter in einem eher bedauernden Ton:] dann hab ich mir so viel Mühe, umsonst Mühe_—“Frau C. unterbricht sie und sagt: „Aber ist doch witzig.“ [-] Dann fragt Frau C. in die Klasse: „Gibt's Tipps?“

Für die Feedbackrunden gibt die Lehrerin eine Phasenstruktur vor, die der Klasse aus früheren Unterrichtsstunden bekannt ist: Auf die Nachfragen an die Präsentierenden folgen die „Tops“ und danach die Tipps aus dem Plenum, bevor die Noten festgelegt werden. Die Lehrerin leitet von einer Phase zur nächsten über, ansonsten wird das Rederecht in der Phase der Rückmeldungen offiziell von den Präsentierenden erteilt. Mit dieser Selektionsmöglichkeit können sie beeinflussen, wessen Eindrücke zuerst gehört werden und damit womöglich die Stoßrichtung für weitere Beiträge vorgeben.

Das Selektionsrecht umfasst auch eine Verpflichtung, die sie nicht delegieren dürfen, wie sich an der Interaktion zwischen Pia und den beiden Luises zeigt. Frau C. beansprucht für sich das Recht, Pia zu unterbrechen, ebenso scheint es nicht sanktionswürdig zu sein, wenn die Präsentierenden Aussagen ihrer Mitschülerinnen oder Mitschüler unterbrechen, um anderen das Wort zu erteilen.

Die Interventionen der Lehrerin zeigen, dass eine Stellungnahme der Präsentierenden unerwünscht ist. Tim wird verwehrt, den von ihm anscheinend subjektiv als höher bewerteten Anspruch des Selbstzeichnens zur Geltung zu bringen, obwohl dies Einfluss auf die Notenfindung haben könnte. Gleichzeitig macht Frau C. Tim dezent, aber klassenöffentlich auf eine Regelverletzung aufmerksam, die sich in den kommenden Minuten steigernd wiederholen wird: Er soll nicht auf das Feedback antworten. Es ist kein Raum vorgesehen, um die Wahrnehmung der gezeigten Leistung zu verändern oder die eigene Leistung zu reflektieren. In der nächsten Phase soll das Plenum konstruktive Kritik äußern.

Erneut wird Chris aufgerufen, er meint: „Ich finde, du [Tim] hättest, also ein bisschen mehr zum Publikum schauen.“ Dieser ruft laut: „Ich musste *unverständlich* [Inhaltlich: ablesen].“

Die Lehrerin nimmt nun Mira dran, die sagt: „Ähm, ich hab gleich zwei. Ähm ich fand, dass ihr ein bisschen sehr viel verraten habt, also, also das war ziemlich viel, was ihr da vorgelesen habt, also-_"

Frau C. unterbricht und sagt kurz: „Die Beschreibung, meinste?_-“, Tim unterbricht wiederum Frau C. und sagt laut: „Ich wollte vier Seiten VORLESEN, weil das war für mich halt das SPANNEndste. “ Die Lehrerin macht nur „Mmh.“ und Mira, die mit ihrer Antwort noch nicht fertig war, will nun ihren zweiten Tipp anführen: ,und ähm, ähm, ich fand auch bei—“ Doch sie wird erneut unterbrochen, dieses Mal jedoch von Pia: „Ich hab noch nicht den Verlag gesagt.“ „Doch, Verlag Loewe.“ ruft jemand aus der Klasse rein, die Lehrerin nickt. 
Gleich den ersten Kritikpunkt versucht Tim mit einer Erklärung seines Vorgehens zu entkräften. Die Lehrerin geht darauf nicht explizit ein, entzieht beiden aber vorübergehend das Selektionsrecht, indem sie selbst Mira aufruft, deren Aussagen sie mit einer klärenden Nachfrage unterbricht, wodurch sie sich als wahrgenommene Adressatin positioniert. Das Selektionsrecht der Lehrerin qua Hierarchie schlägt hier das Selektionsrecht der Präsentierenden qua Methodenwahl. Tim seinerseits unterbricht Frau C., um auch auf Miras Kritik verteidigend zu reagieren. Mira, die von Pia unterbrochen wird, kann ihren zweiten Tipp nicht mehr anbringen. Ein Hereinrufen wird von Frau C. nickend aufgegriffen und damit akzeptiert.

Tim ruft nun Jasper auf, der sagt: „Ähm, ich find, dass du immer Pia was abgefragt hast [inhaltlich: Das Interview zu Beginn], das-“ “Frau C. unterbricht Jasper: „Das hat dir NIIICHT gefallen?“

Jasper: „Ja, weil er immer Pia, Pia, Pia.“

Frau C: „Ok. Aber das Prinzip fandste gut, dass er Fragen gestellt hat, nur er hätte anders formulieren sollen. Alles klar.“ Während Jasper im Hintergrund leise weiter redet, nennt Tim schon Marlons Namen.

Marlon: „Ich wollte noch was zu den Tops sagen. Ich fand es gut, dass wenn ihr mal ähm, ihr habt mich ja gefragt, weil ihr nicht wusstet, wo der Verlag und der Autor, fand ich es gut, dass ihr euch Hilfe geholt habt.“"

Die Lehrerin macht wieder ein bestätigendes „Mhh“ und sagt: „Genau, so muss das-“ “

Tim unterbricht Frau C. und sagt: „Ähm, ich hab noch eins vergessen, die Hauptfan-figuren sind Anne und Phillipp [Frau C.: „Das haben wir aber verstanden“] und Slim Cool [Frau C.: „Haben wir verstanden."] und Charly.“

Jemand ruft unaufgefordert hinein, dass Pia weniger gesprochen habe. Die Lehrerin bestätigt das (,Ja, das stimmt.“) und Tim steigt erneut ein: „Naja, sie hat-“ "Die Lehrerin lässt Tim nun nicht weiterreden und sagt in einem lauten Flüsterton:

„TIM, einfach hinnehmen. Nicht immer rechtfertigen, das ist, das was so (.) angekommen ist. Du musst das nicht noch begründen. OKAY. [Dann blickt sie wieder in die Klassen und spricht normal weiter] Note?“”

Frau C. greift nun stärker moderierend ein, obwohl dies eigentlich den Präsentierenden obliegt. Als Jasper den Interview-Stil kritisiert, der im Wortbeitrag einer Schülerin zuvor gelobt und von Frau C. als gut bestätigt worden war, fragt sie nach, ob ihm das nicht gefallen habe. Die Betonung des Wortes ,nicht“" transportiert zusammen mit Stimmlage und Intonation den Zweifel der Lehrerin an der Korrektheit der Aussage. Jasper erhält die Möglichkeit, seinen Beitrag zu korrigieren bzw. zu konkretisieren und nutzt sie, um auf die aus seiner Sicht stilistisch ungeschickte Dopplung zu verweisen. Frau C. formuliert seine Aussage um und liest sie so, dass er die Idee grundsätzlich gut, nur nicht optimal umgesetzt fand. Dies bleibt als Ergebnis stehen, ohne dass Jasper es als korrekte Lesart bestätigt hätte.

Anschließend durchbricht Marlon ungerügt den vorgegebenen Phasenablauf. In seinem Lob steckt eine sprachlich elaborierte Selbstpositionierung, denn Marlon hebt nicht nur hervor, dass sich die Präsentierenden bei ihm Hilfe geholt haben, sondern er weist sich selbst als Mitwirkender an der erbrachten Leistung aus. Tim 
geht nicht darauf ein, sondern fährt eine doppelte Reparaturstrategie: Zum einen setzt er Marlons Anteil an der Leistung nun seinen eigenen Anteil entgegen, zum anderen versucht er noch einmal, die von Frau C. geforderten Eckpunkte aufzugreifen.

Erneut betrachtet Frau C. solche Praktiken aber als Regelverletzungen. Ihr wiederholtes ,(Das) Haben wir verstanden“ zeigt, dass Tim die von ihr gesetzte Form stört: Er darf (mit-)aussuchen, wer sprechen darf, aber den Rückmeldungen nichts hinzufügen. Mit dem verkollektivierenden „Wir“, mit dem sie gleichzeitig ein „Du“ bzw. „Ihr beide“ konstruiert, spricht Frau C. auch für die restliche Klasse. Eine Gegenpositionierung der anderen Schülerinnen und Schüler ist kaum mehr möglich.

Als die nächste Kritik unaufgefordert hereingerufen wird, reagiert die Lehrerin bestätigend und markiert so als akzeptabel, dass das Selektionsrecht der Präsentierenden nicht nur von ihr, sondern auch vom Rest der Klasse untergraben wird. Gleichzeitig positioniert sie sich damit als jemand, der - im Gegensatz zu den beiden Präsentierenden - in der Lage ist, Kritik einzuschätzen sowie zu bestätigen und zurückzuweisen. Als Tim wieder verteidigend reagieren möchte, artikuliert Frau C., was sie vorher latent zu vermitteln versucht hat: Er solle die Beurteilung ,einfach hinnehmen“. Da die Ethnographin diese Aussagen hören kann, ist der Flüsterton nicht nur als Adressierung Tims zu verstehen, obwohl das Flüstern suggerieren könnte, dass es sich um eine persönliche Interaktion handelt. Es lenkt die Aufmerksamkeit der Klasse auf die eigentlich dyadische Interaktion. Nicht nur Tim soll die Regeln des Formats lernen, sondern auch die anderen Schülerinnen und Schüler: Sie werden an die Regeln erinnert und überdies als Publikum des Verweises Teil der Sanktion, die ihnen gleichzeitig Frau C.s Umgang mit Regelverletzungen demonstriert, der sich ebenso auf sie auswirken könnte.

Anschließend leitet die Lehrerin in die letzte Phase, die Notenfindung, über.

Frau C. ruft nun Marlon auf, der daraufhin eher fragend sagt: „Zwei?“ Jemand anderes sagt zeitgleich sicherer „Eins.“ Frau C. nennt noch einmal Marlons Namen.

Marlon: „Präsentation würde ich ne 1 geben.“

Frau C.: „Mmh. (...) Wenn ihr das vergleicht mit dem, was IHR gemacht habt, was Marlene und Chris gemacht haben, was die Luise mit Mira gemacht. War das das gleiche Niveau?" Es ist nun auf einmal sehr still und die meisten Kinder schauen nach vorne. Vermutlich Marlon antwortet leise: „Also, ich find, dass Gebastelte (.) find ich (.) auch gut, (.) ähm das, aber das für das Erzählen würde ich wirklich ne Eins geben, weil-_“

Frau C. unterbricht ihn und sagt: „Für das WIE? [leises „Ja“ von Marlon, zeitgleich] Das ja. Aber das, WAS sie euch präsentiert haben?“

Jetzt ruft Tim energischer: „Wir HABen noch eine Sache, noch ein—“ Doch die Lehrerin geht nicht darauf ein und sagt: „Ricardo.“ Er (zunächst laut, dann immer leiser werdend) sagt: „Also ich finde ähm, für die Präsentation dafür auch ne Eins, aber ähm für das (.) für das andere ne Zwei.“

Frau C. schnell: „DAS mein ich auch, ne. [Jemand ruft: „Ich auch“.] Wenn ihr das ins, ins Verhältnis setzt-““

Anders als in den Phasen zuvor gewährt Frau C. das Selektionsrecht nicht mehr den Präsentierenden, sondern ruft selbst Schülerinnen und Schüler auf. Marlon, 
der unsicher eine Note vorschlägt, modifiziert seine Antwort, die dennoch nicht die zu sein scheint, die Frau C. hören will. Frau C.s Anregung, die Leistung im sozialen Vergleich zu betrachten, setzt die soziale Bezugsnorm (vgl. Rheinberg und Fries 2018) als entscheidend und lässt Marlons Vorschlag korrekturbedürftig wirken. Alternativ hätte sie zum Begründen und Vergleichen auffordern können, da Marlon nicht präzisiert hat, was er besonders gelungen fand. Sein Vorgehen lässt sich als Absicherungsstrategie interpretieren: Er, der ursprünglich eine Zwei vorgeschlagen hatte, bleibt nun bei seiner Eins, stimmt aber auch der Zwei (und damit Frau C.) zu jedoch in einem anderen Teilbereich. Er positioniert sich als jemand, der berechtigt an der Notenvergabe partizipieren kann, denn seine Einschätzung ist nicht falsch, sondern nur nicht differenziert genug. Die Zensurenvergabe basiert nämlich auf zwei Noten: dem Vortrag(sstil) und dem Gebastelten.

Erneut versucht Tim einzugreifen, was Frau C. jedoch ignoriert, indem sie mit der Notenfindung fortfährt, dabei weiter- und den nächsten Schüler ansprechend. Dieser bestätigt die von Marlon abgegebene Bewertung deutlicher und nennt die Antwort, auf die Frau C. ihrer Reaktion zufolge gewartet hat. Sie bestätigt die Bewertung und setzt dazu an, ihre Begründung auszuführen und erneut auf einen Vergleich hinzuweisen, ohne diesen zu konkretisieren. Doch noch sehen Pia und Tim Spielraum.

Pia unterbricht und ruft, dass sie doch aber was vergessen haben. Die Lehrerin redet weiter: „Ja, passiert [Tim unterbricht, Frau $\mathrm{C}$ redet weiter, beide reden gleichzeitig, Tim: „Wir haben noch ein-“], aber wir müssen ja das bewerten, was wir sehen, Pia, ne? Wir können ja nicht sagen, eigentlich ist es gut gedacht, AAABER. (..) Ok."

Es ist jetzt ruhiger, auch im hinteren Kreis der Klasse. Diese Ruhe nutzt Tim, um endlich zu sagen: ,Wir haben noch eine Sache, ein Plakat, haben wir noch. Da wollten wir „ABENDrot und Anne“ aber zeigen.“

Frau C: „Ja, ihr habt auch, ihr habt ganz, ganz viel angefangen, ganz viel gute Ideen, dann habt ihr euch so ein bisschen verzettelt, ne? Und (Tim ruft laut: „Ja, dann-“) dadurch seid ihr dann nicht mehr auf den Punkt gekommen."

Tim redet weiter: „Dann haben wir halt zu Hause was ganz anderes gemacht. [Frau C: „Ja, genau.“] Und die Eltern nicht wussten, WAS wir alles machen sollten, dann haben die Autor, Verlag und so, alles vergessen, das hab ich-““

Frau C unterbricht und sagt: ,Zu den ELTERN? Was haben DIE denn damit zu tun, ihr sollt das machen. [Tim unterbricht: „Die haben uns geholfen.“] Ja, aber sollen sie ja nicht. IHR solltet das ja machen. [Pia quetscht ein leises „Ja“ raus.] $[-] . “$

Tim: „Also die haben uns Tipps gegeben.“

„Okay, Tipps geben, ja, das ist in Ordnung, aber eigentlich ist es eure Aufgabe. [Frau $\mathrm{C}$ spricht in einem anderen Ton, eher wieder laut-flüsternd weiter:] LERNT dazu. Wenn ihr nächstes Mal was anfangt, nicht mit TAUSEND verschiedenen Dingen anfangen, weil ihr so viele gute Ideen habt, sondern miteinander komm-sprechen und sich auf EINE festlegen und DIE durchziehen. Na? [-] Klar? (Keine Antwort von den beiden.) Okay. [Frau C redet laut/normal weiter:] Dann brauchen wir noch Ricardo und Johann.“ 
Beide Korrekturversuche von Pia und Tim weist Frau C. mit einer Wir-Formulierung zurück. Sie führt dazu die Begründung an, dass nur die sichtbare Leistung bewertet werden könne. Erneut gelingt es Tim, sich soweit zu behaupten, dass er auf die zusätzlichen Elemente seiner und Pias Leistung aufmerksam machen kann. Diese wiederholte Regelverletzung beantwortet Frau C. mit Einwänden, die allerdings nicht auf einer Metaebene (im Sinne von „Die Note bezieht sich darauf, was ihr vorhin gezeigt habt.“), sondern auf inhaltlicher Ebene verortet sind. Damit stellt sie sich außerhalb des Geltungsbereichs des von ihr vorgegebenen Phasenablaufs. Ihr Kritikpunkt bleibt als Ergebnis stehen und gilt nicht als einer von mehreren.

Als Tim sich weiter erklären will, entspinnt sich eine Diskussion, inwiefern das Einholen elterlicher Hilfe legitim ist. Da Schulleistung als persönlich zurechenbar konstruiert wird (vgl. Ricken 2018, S. 54), erfordert dies zusätzliche Legitimierungspraktiken, um die bereits verkündete Bewertung als gültig beibehalten zu können. Frau C.s einlenkendes Zulassen elterlichen Rats rahmt die Benotung als kulant und setzt sie damit in einen Raum des nicht Einklagbaren (vgl. Meier 2011, S. 121). Die zentrale Botschaft an die Klasse, die Frau C. Tim stellvertretend für die Klasse im pseudo-exklusiven lauten Flüsterton vermittelt, ist, dass Schülerinnen und Schüler fremde Einschätzungen ihrer Leistung ,einfach hinnehmen“ müssen, ohne sie zu kommentieren oder gar zu kritisieren. Die Situation der Leistungsbewertung endet mit Frau C.s Fazit, das den aus ihrer Sicht zentralen Schwachpunkt der Präsentation offenlegt, ohne auf die sonst geäußerten Stärken und Schwächen zurückzukommen.

\section{Zusammenfassung: ,doing student' while ,doing participation`}

Die Lehrerin versucht, die Prüfungssituation so zu gestalten, dass die Note legitimiert erscheint, wozu neben dem transparenten Verfahrensablauf (Meier 2011, S. 83) beiträgt, dass Mitschülerinnen und Mitschüler in die Besprechung einbezogen werden und die Note aussprechen (ebd., S. 106). Ähnlich wie in der von Meier (2011) skizzierten Leistungsbewertungsepisode kommt es ,,[d]en Schülern (...) in diesem Schauspiel zu, brav mitzuspielen“ (S. 96), denn Ablauf, Struktur und Verlauf werden von Frau C. gelenkt, so dass die Sequenz Züge einer Pseudo-Partizipation trägt.

Zentral ist die Verschränkung von ,doing student' und ,doing participation ', bei der die Anerkennung heteronomer Normen Voraussetzung ist für die gewährten Autonomieräume. Während offiziell die erbrachte Leistung in Form der Buchpräsentation (erste Ebene) zu bewerten ist, werden mit den Rückmeldungen auf einer zweiten Ebene ebenfalls Leistungsdarstellungen aufgeführt. Praktiken der Aufführung und der Bewertung von Leistung (Idel und Rabenstein 2016, S. 12) überlagern einander also in der Situation und die Bewertung von Leistungen wird selbst zu einem Teil einer Leistungsaufführung. Dabei partizipiert die Klasse als „Publikum“ (Breidenstein und Kelle 2002, S. 321) an der Leistungsbewertung. Durch die aufgerufene Leistungsordnung, mit der ,,je spezifische Möglichkeitsräume, Leistung zu zeigen und zu bewerten“ (Idel und Rabenstein 2016, S. 11) einhergehen, interferieren die situationsüberdauernden Normen des ,doing student' die situationsspezifischen Normen des, doing participation“. Exemplarisch verdeutlicht Marlons Äußerung, wie ,doing participation'-Praktiken als Teil von ,doing student` zur Selbstpositionierung 
genutzt werden können. Subjektivierungstheoretisch zeigt sich, wie die Schülerinnen und Schüler die Leistungsnormen reproduzieren, wobei nach der „Logik der (Prüfungs-Praktiken)“ (Ricken 2018, S. 54) die gezeigte Leistung immer mit den Fähigkeiten des Subjekts verbunden wird. Durch den Vollzug der interdependenten ,doing participation'- und ,doing student'-Praktiken wird eine situative, wirkmächtige Ordnung etabliert.

Das Format suggeriert den Schülerinnen und Schülern im Plenum, dass sie in der Lage seien, die Leistung ihrer Mitschülerinnen und Mitschüler angemessen zu bewerten. Das Plenum partizipiert formal an der Bewertungsmacht der Lehrerin, wird dabei aber (zwangs-)distanziert von den Präsentierenden. So wird zwar zu Tim und Pia gesprochen, aber nicht mit ihnen. Die Nutzung der gewährten Freiräume orientiert sich an den Erwartungen schulischer Leistungsdarstellung.

Die Partizipationsmöglichkeiten von Tim und Pia unterscheiden sich von denen der anderen Schülerinnen und Schüler und verändern sich im Verlauf der Situation. Auffällig sind die Grenzziehungen und das Zurückweisen von Tims Korrekturversuchen, die sich in verbalen wie nonverbalen Praktiken der Lehrerin zeigen: Von der kopfschüttelnden Verstummungsaufforderung bis zur Maßregelung im lauten Flüsterton macht Frau C. wiederholt Tims Normbrüche deutlich. Während die Lehrerin den Vortrag als abgeschlossene Leistung verstanden wissen will, den es (fremd) zu evaluieren gilt, scheint diese Abgeschlossenheit für Tim nicht nachvollziehbar oder akzeptabel. Obwohl Normen verändert werden können (Butler 2001), gelingt Tim dies nicht. Seine mehrfachen Selbstermächtigungsversuche scheitern, er kann sich nicht als gleichberechtigter Interaktionspartner neben der Lehrerin positionieren.

Spiegelbildlich zeigen sich auch bei Frau C. die zwei Normensets, die ineinander verstrickt wirksam werden. Die über das Format initiierten Praktiken des, doing moderator" werden überlagert von den tendenziell situationsüberdauernden Praktiken des , doing teacher'. Wo die Lehrerin die Partizipation strukturiert, indem sie die Einhaltung des formalen Ablaufs unterstützt, kann das zu den pädagogischen ,,Stützungen“ (Helsper und Lingkost 2013, S. 134) zählen und als Teil des ,doing moderator betrachtet werden. Wo sie aber den Inhalt des Feedbacks reguliert, inhaltlich auf das angestrebte Ergebnis zusteuert, Wortbeiträge sinnändernd (um)deutet und nur die weiterverfolgt, die hinsichtlich ihrer Notenvorstellung sinnvoll scheinen, nutzt sie Praktiken des ,doing teacher', die an gelenkte Unterrichtsgespräche erinnern.

Dabei setzt sie den Äußerungen der Schülerinnen und Schüler keine Ich-Botschaften entgegen, mit denen sie sich als gleichberechtigt im Bewertungsprozess positionieren könnte. Sie drängt nicht auf stichhaltige Argumente, mit denen unterschiedliche Positionen gegeneinander abgewogen werden könnten. Ihre Reaktionen verweisen darauf, dass es um das Finden des, richtigen“ Ergebnisses geht, das die Lehrerin (aner-)kennt. Vergleichbar beschreibt Meier (2011, S. 106), wie Objektivität für eine Benotung beansprucht wird, deren Legitimität unter anderem ,durch das Herausfinden der richtigen Note einer vermeintlich unabhängigen Schülerin situativ hergestellt" werde.

Wer stabilisierend am Format mitwirkt, kann sich zwar äußern, der individuellen Aussage wird jedoch inhaltlich eine geringe Relevanz beigemessen. So können sich die Schülerinnen und Schüler beispielsweise ungeahndet gegenseitig unterbrechen. Würde dieses Unterbrechen durch Aufrufen des nächsten Gruppenmitglieds bei in- 
dividualistischer Deutung einen Affront darstellen, wird es in der kollektivistischen Logik verständlich: Jede Schülerin und jeder Schüler spricht stellvertretend für die Gruppe, die als Kollektiv dem ,richtigen ' Ergebnis näherkommt, für das es einen angeblichen Konsens in der Gruppe der beurteilenden Schülerinnen und Schüler gibt. Als die Schülerinnen und Schüler am Gespräch zur Notenfindung beteiligt werden, wirkt dies nicht ermächtigend, sondern führt vor Augen, dass sie sich der Lehrerin unterordnen müssen, ihrer Macht selbst kollektiv nichts entgegensetzen können, wodurch die asymmetrische Ordnung stabilisiert wird. Frau C. hat ihre Position nur scheinbar geschwächt, indem sie offiziell die Klasse bewerten lässt. Verschiedene Führungs- und Sanktionierungspraktiken, beispielsweise, wie sie Tims kommunikative Widerständigkeit klassenöffentlich und stellvertretend als Normbruch adressiert, stärken vielmehr ihre Position. Die Schülerinnen und Schüler können und sollen partizipieren, als heteronomes Publikum können sie sich der Situation nicht entziehen und sind für die Hervorbringung der Ordnung ein relevanter Teil der Situation. Sie haben jedoch kaum Chancen, das Ergebnis zu beeinflussen: Sie werden als Teil der Gruppe zum Sprachrohr des Benotungsvorgangs der Lehrerin und damit als aktive Claqueure zur Legitimation der Bewertung instrumentalisiert.

Die Partizipation der Schülerinnen und Schüler wirkt, nach Helsper und Lingkost (2013), verordnet, wo zur Beteiligung gedrängt wird, instrumentalisiert, wo Aussagen umgedeutet werden, um auf das gewünschte Ergebnis hinzuführen, kontrollförmig, wo Schülerinnen und Schüler zu Gehilfen der Lehrerin gemacht werden oder simuliert, falls man unterstellt, dass die Entscheidung für eine bestimmte Note schon vor den Beiträgen aus dem Plenum gefallen war. Im Partizipieren lernen Schülerinnen und Schüler in dieser Szene am Beispiel des Zustandekommens einer Benotung, wie Personen, die aufgrund der Umstände den Gang der Entscheidung kaum beeinflussen können, hinterher dennoch das Ergebnis (mit) zu verantworten haben. Das potenziell ermächtigende Element, über die eigene Note diskutieren zu können, schließt die Lehrerin aus und die Entscheidung sollen die Schülerinnen und Schüler ,einfach hinnehmen“. Gefordert wird also eine Akzeptanz fremder Beschlüsse als Ergebnisse eines Verfahrens, das nicht zur Debatte steht und dennoch wirkmächtiger ist als alle eröffneten Äußerungsmöglichkeiten.

,Doing participation` zeigt sich hier eng verschränkt mit ,doing student` und ,doing teacher", denn es ist eine Partizipation als Schülerin bzw. Schüler, bei der die Grenzen des Sag- und Machbaren bestimmt werden von der Adressierung als Teil einer Gruppe (des Plenums) sowie von den antizipierten Intentionen der Lehrperson und nicht von den Ansprüchen einer inhaltlichen Rationalität. Da die unterrichtliche Ordnung eine Leistungsordnung ist (Rabenstein et al. 2013) und potenziell alles, was im Unterricht getan wird, in die Leistungsbewertung eingeht (Breidenstein 2006, S. $261 \mathrm{f}$.), führt die Aufgabe der Partizipation für Schülerinnen und Schüler zu einer Situation, in der sie sich an Normen schulischer Leistungserbringung orientieren müssen. Es griffe daher zu kurz, die Analyse als Einzelfall zu deuten, denn die rekonstruierten Logiken des Feldes (vgl. Breidenstein et al. 2020) verweisen auf ein Dilemma der Partizipationsbestrebungen im Unterricht: Wenn ,doing student ' dem ,doing participation' widerspricht, ist nicht zu erwarten, dass sich Praktiken einüben lassen, die den Ansprüchen gerecht werden, die an Partizipation gestellt werden. Die Widersprüchlichkeit der Normen zu ignorieren, hieße die Zwänge, in denen 
sich Schülerinnen und Schüler bewegen, zu ignorieren und „Partizipationsplacebo[s]“ (Hedtke 2016, S. 6) zu fördern. Konzepte partizipativer Leistungsbewertung empirisch daraufhin zu untersuchen, inwiefern sie unter ,doing student“-Bedingungen umsetzbar sind, und die Erkenntnisse in didaktische Weiterentwicklungen einzubeziehen, wäre vor diesem Hintergrund eine Aufgabe zukünftiger Schul- und Unterrichtsforschung.

Funding Open Access funding enabled and organized by Projekt DEAL.

Open Access Dieser Artikel wird unter der Creative Commons Namensnennung 4.0 International Lizenz veröffentlicht, welche die Nutzung, Vervielfältigung, Bearbeitung, Verbreitung und Wiedergabe in jeglichem Medium und Format erlaubt, sofern Sie den/die ursprünglichen Autor(en) und die Quelle ordnungsgemäß nennen, einen Link zur Creative Commons Lizenz beifügen und angeben, ob Änderungen vorgenommen wurden.

Die in diesem Artikel enthaltenen Bilder und sonstiges Drittmaterial unterliegen ebenfalls der genannten Creative Commons Lizenz, sofern sich aus der Abbildungslegende nichts anderes ergibt. Sofern das betreffende Material nicht unter der genannten Creative Commons Lizenz steht und die betreffende Handlung nicht nach gesetzlichen Vorschriften erlaubt ist, ist für die oben aufgeführten Weiterverwendungen des Materials die Einwilligung des jeweiligen Rechteinhabers einzuholen.

Weitere Details zur Lizenz entnehmen Sie bitte der Lizenzinformation auf http://creativecommons.org/ licenses/by/4.0/deed.de.

\section{Literatur}

Abs, H. J., \& Moldenhauer, A. (2021). Partizipation in Schule und Unterricht. In T. Hascher, T.-I. Idel \& W. Helsper (Hrsg.), Handbuch Schulforschung (S. 1-19). Wiesbaden: VS.

Betz, T., Gaiser, W., \& Pluto, L. (2010). Partizipation von Kindern und Jugendlichen. In T. Betz, W. Gaiser \& L. Pluto (Hrsg.), Partizipation von Kindern und Jugendlichen. Forschungsergebnisse, Bewertungen, Handlungsmöglichkeiten (S. 11-31). Schwalbach: Wochenschau.

Beutel, S.-I., \& Pant, H. A. (2020). Lernen ohne Noten. Alternative Konzepte der Leistungsbeurteilung. Stuttgart: Kohlhammer.

Billis, J. (2020). Partizipation. In P. Bollweg, J. Buchna, T. Coelen \& H.-U. Otto (Hrsg.), Handbuch Ganztagsbildung (2. Aufl. S. 367-378). Wiesbaden: Springer VS.

Breidenstein, G. (2004). KlassenRäume - eine Analyse räumlicher Bedingungen und Effekte des Schülerhandelns. Zeitschrift für qualitative Bildungs-, Beratungs- und Sozialforschung, 5(1), 87-107.

Breidenstein, G. (2006). Teilnahme am Unterricht. Ethnographische Studien zum Schülerjob. Wiesbaden: VS.

Breidenstein, G., \& Kelle, H. (2002). Die Schulklasse als Publikum. Zum Verhältnis von Peer Culture und Unterricht. Die deutsche Schule, 94(3), 318-329.

Breidenstein, G., Hirschauer, S., Kalthoff, H., \& Nieswand, B. (2020). Ethnografie. Die Praxis der Feldforschung (3. Aufl.). München: UVK.

Breidenstein, G., Meier, M., \& Zaborowski, K. U. (2011). Das Projekt Leistungsbewertung in der Schulklasse. In K. U. Zaborowski, M. Meier \& G. Breidenstein (Hrsg.), Leistungsbewertung und Unterricht. Ethnographische Studien zur Bewertungspraxis in Gymnasium und Sekundarschule (S. 15-37). Wiesbaden: VS.

Budde, J. (2010). Inszenierte Mitbestimmung?! Soziale und demokratische Kompetenzen im schulischen Alltag. Zeitschrift für Pädagogik, 56(3), 384-401.

Budde, J. (2014). Differenz beobachten? In A. Tervooren, N. Engel, M. Göhlich, I. Miethe \& S. Reh (Hrsg.), Ethnographie und Differenz in pädagogischen Feldern. Intentionale Entwicklungen erziehungswissenschaftlicher Forschung (S. 133-148). Bielefeld: transcript.

Butler, J. (1998). Haß spricht. Zur Politik des Performativen. Frankfurt a.M.: Suhrkamp.

Butler, J. (2001). Psyche der Macht. Das Subjekt der Unterwerfung. Frankfurt a.M.: Suhrkamp. 
Butler, J. (2002). Ein Interview mit Judith Butler. In H. Bublitz (Hrsg.), Judith Butler zur Einführung (S. 142-152). Hamburg: Junius.

Coelen, T.W. (2010). Partizipation und Demokratiebildung in pädagogischen Institutionen. Zeitschrift für Pädagogik, 56(1), 37-52.

Feichter, H.J. (2020). Die Grammatik der Schule als Partizipationshindernis. Organisationstheoretische und schulkulturelle Überlegungen. In S. Gerhartz-Reiter \& C. Reisenauer (Hrsg.), Partizipation und Schule (S. 25-40). Wiesbaden: VS.

Gamsjäger, M., Langer, R., \& Altrichter, H. (2013). Schulentwicklung durch Partizipation von SchülervertreterInnen? In S. M. Weber, M. Göhlich, A. Schröer, C. Fahrenwald \& H. Macha (Hrsg.), Organisation und Partizipation. Beiträge der Kommission Organisationspädagogik (S. 149-156). Wiesbaden: VS.

Gruschka, A. (2003). Von der Kritik zur Konstruktion ist es oft nur ein Schritt: der der Negation. Pädagogische Korrespondenz, 30(03), 71-79.

Häcker, T. (2011). Portfolio revisited - über Grenzen und Möglichkeiten eines vielversprechenden Konzepts. In T. Meyer, K. Mayrberger, S. Münte-Goussar \& C. Schwalbe (Hrsg.), Kontrolle und Selbstkontrolle. Zur Ambivalenz von E-Portfolios in Bildungsprozessen (S. 161-183). Wiesbaden: VS.

Hahn-Laudenberg, K. (2017). Konzepte von Demokratie bei Schülerinnen und Schülern. Wiesbaden: VS.

Hart, R. A. (1992). Children's participation: from tokenism to citizenship. Florence: UNICEF.

Hedtke, R. (2016). Bildung zur Partizipation: Funktionalismus, Pädagogisierung oder Politisierung? Universität Bielefeld, Fakultät für Soziologie, Working Paper No. 4. https://pub.uni-bielefeld.de/ download/2905352/2905354/neu_Hedtke_WP_4_2014_bildung-zur-partizipation.pdf. Zugegriffen: 13. März 2021.

Helsper, W., \& Lingkost, A. (2013). Schülerpartizipation in den Antinomien von Autonomie und Zwang sowie Organisation und Interaktion - exemplarische Rekonstruktion im Horizont einer Theorie schulischer Anerkennung. In B. Hafeneger, P. Henkenborg \& A. Scherr (Hrsg.), Pädagogik der Anerkennung. Grundlagen, Konzepte, Praxisfelder (S. 132-156). Schwalbach: Wochenschau.

Idel, T.-S., \& Rabenstein, K. (2016). Leistung als soziale Konstruktion: Was müssen Schüler/-innen wissen und können, um im heterogenitätssensiblen individualisierenden Unterricht Anerkennung zu finden? In S. Doff (Hrsg.), Heterogenität im Fremdsprachenunterricht (S. 9-19). Tübingen: Narr Francke Attempto.

KMK (2018). Demokratie als Ziel, Gegenstand und Praxis historisch-politischer Bildung und Erziehung in der Schule. Beschluss der Kultusministerkonferenz vom 06.03.2009 i.d. F. vom 11.10.2018

Knauer, R., Hansen, R., \& Sturzenhecker, B. (2016). Demokratische Partizipation in Kindertageseinrichtungen. Konzeptionelle Grundlagen. In R. Knauer \& B. Sturzenhecker (Hrsg.), Demokratische Partizipation von Kindern (S. 31-46). Weinheim: Beltz Juventa.

Maywald, J. (2016). Das Recht gehört zu werden. Beteiligung als Grundrecht jedes Kindes. In R. Knauer \& B. Sturzenhecker (Hrsg.), Demokratische Partizipation von Kindern (S. 16-30). Weinheim, Basel: Beltz Juventa.

Meier, M. (2011). Die Praktiken des Schulerfolgs. In K. U. Zaborowski, M. Meier \& G. Breidenstein (Hrsg.), Leistungsbewertung und Unterricht. Ethnographische Studien zur Bewertungspraxis in Gymnasium und Sekundarschule (S. 39-161). Wiesbaden: VS.

Pigorsch, S., \& Rothmaler, J. (2020). Von Essensplänen und „Super-Bürgern“. Ein Feldvergleich von Beteiligungspraktiken in Kita und Stadt. In S. Thomas \& J. Rothmaler (Hrsg.), Partizipation in der Bildungsforschung (S. 224-250). Weinheim: Beltz Juventa.

Rabenstein, K., Reh, S., Ricken, N., \& Idel, T.-S. (2013). Ethnographie pädagogischer Differenzordnungen. Methodologische Probleme einer ethnographischen Erforschung der sozial selektiven Herstellung von Schulerfolg im Unterricht. Zeitschrift für Pädagogik, 89(5), 668-690.

Reckwitz, A. (2008). Unscharfe Grenzen. Perspektiven einer Kultursoziologie. Bielefeld: transcript.

Reichenbach, R. (2006). Diskurse zwischen Ungleichen. Zur Ambivalenz einer partizipativen Pädagogik. In C. Quesel \& F. Oser (Hrsg.), Die Mühen der Freiheit. Probleme und Chancen der Partizipation von Kindern und Jugendlichen (S. 39-61). Zürich, Chur: Rüegger.

Reinhardt, V. (2010). Kriterien für eine demokratische Schulqualität. In D. Lange \& G. Himmelmann (Hrsg.), Demokratiedidaktik. Impulse für die politische Bildung (S. 86-102). Wiesbaden: VS.

Reisenauer, C. (2020). Kinder- und Jugendpartizipation im schulischen Feld - 7 Facetten eines vielversprechenden Begriffs. In S. Gerhartz-Reiter \& C. Reisenauer (Hrsg.), Partizipation und Schule (S. 3-22). Wiesbaden: VS.

Rheinberg, F., \& Fries, S. (2018). Bezugsnormorientierung. In D. Rost, J. R. Sparfeldt \& S. R. Buch (Hrsg.), Handwörterbuch Pädagogische Psychologie (5. Aufl. S. 56-63). Weinheim: Beltz. 
Ricken, N. (2018). Konstruktionen der ,Leistung‘. Zur (Subjektivierungs-)Logik eines Konzepts. In S. Reh \& N. Ricken (Hrsg.), Leistung als Paradigma. Zur Entstehung und Transformation eines pädagogischen Konzepts (S. 43-60). Wiesbaden: VS.

Sauerwein, M.N. (2019). Partizipation in der Ganztagsschule - vertiefende Analysen. Zeitschrift für Erziehungswissenschaft, 22(2), 435-459.

Schäfer, H. (2013). Die Instabilität der Praxis. Reproduktion und Transformation des Sozialen in der Praxistheorie. Weilerswist: Velbrück Wissenschaft. 